\title{
Singlet-Triplet Mixing in Superconductor-Ferromagnet Hybrid Devices
}

\author{
M. Eschrig ${ }^{1}$, J. Kopu ${ }^{1,2}$, A. Konstandin ${ }^{1}$, J.C. Cuevas ${ }^{1}$, M.Fogelström ${ }^{3}$, and \\ Gerd Schön ${ }^{1,4}$ \\ 1 Institut für Theoretische Festkörperphysik, Universität Karlsruhe, 76128 \\ Karlsruhe, Germany \\ 2 Low Temperature Laboratory, Helsinki University of Technology, FIN-02015 \\ HUT, Finland \\ 3 Applied Quantum Physics, MC2, Chalmers, S-41296 Göteborg, Sweden \\ 4 Institut für Nanotechnologie, Forschungszentrum Karlsruhe, 76021 Karlsruhe, \\ Germany
}

\begin{abstract}
We develop a theory which describes hybrid structures consisting out of superconducting and ferromagnetic parts. We give two examples for applications. First, we consider a hybrid structure containing a strong ferromagnet in the ballistic limit. Second, we study for a weak ferromagnet the influence of a domain wall on the superconducting proximity effect. In both cases we account quantitatively for the mixing between singlet and triplet correlations.
\end{abstract}

\section{Introduction}

Hybrid structures containing ferromagnetic materials became recently a focus of nanoelectronic research because of their relevance for spintronics applications. Consequently, it is desirable to understand how in the case of a superconductor coupled to a ferromagnetic material superconducting correlations penetrate into the ferromagnet. A powerful method to treat such problems is the quasiclassical theory of superconductivity developed by Larkin and Ovchinnikov and by Eilenberger [1,2]. Within this theory the quasiparticle motion is treated on a classical level, whereas the particle-hole and the spin degree of freedoms are treated quantum mechanically. The paper consist of two parts. First we demonstrate our method for a ballistic heterostructure containing a strong ferromagnet, for which we chose for simplification a completely polarized ferromagnet, a half metal. We show results for the modification of the quasiparticle density of states due to the proximity effect in the half-metallic material in a superconductor/half metal/superconductor device (see Fig. 1, left). Second, we study for a diffusive heterostructure containing a weak ferromagnet the influence of a domain wall of the Bloch type on the quasiparticle spectrum. The corresponding setup is shown on the right in Fig. 1. We show results for different ratios between the domain wall width and the superconducting coherence length. 

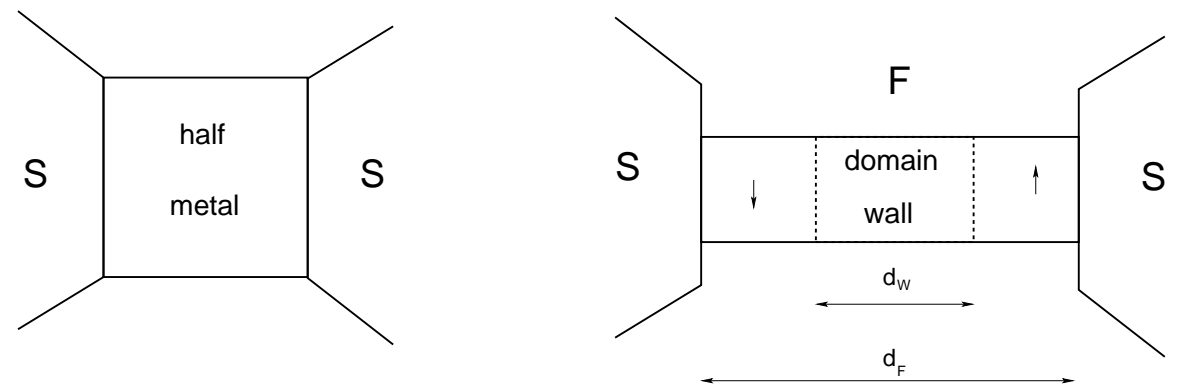

Fig. 1. Schematic picture of the studied devices. On the left, a ballistic heterostructure where a half metal is sandwiched between two superconductors. Here, the conventional proximity effect is completely suppressed as a result of the complete spin polarization of the half metal. On the right, a diffusive weak ferromagnet between two superconductors. The ferromagnet contains a Bloch domain wall of size $d_{W}$

\section{Basic Equations: Ballistic Case}

\subsection{Transport Equations}

The central quantity in quasiclassical theory of superconductivity $[1,2]$ is the quasiclassical Green's function $\breve{g}\left(\mathbf{p}_{F}, \mathbf{R}, E, t\right)$ that depends on the spatial coordinate $\mathbf{R}$ and time $t$. It describes quasiparticles with energy $E$ (measured from the chemical potential) and Fermi momentum $\mathbf{p}_{F}$ moving along classical trajectories with direction given by the Fermi velocity $\mathbf{v}_{F}\left(\mathbf{p}_{F}\right)$. [3] The quasiclassical Green's function is a functional of self energies $\check{\Sigma}\left(\mathbf{p}_{F}, \mathbf{R}, E, t\right)$, which in general include molecular fields, the superconducting order parameter $\Delta\left(\mathbf{p}_{F}, \mathbf{R}, t\right)$, impurity scattering, and external fields. The quantum mechanical degrees of freedom of the quasiparticles show up in the matrix structure of the quasiclassical propagator and the self energies. It is convenient to formulate the theory using $2 \times 2$ matrices in Keldysh space (denoted by a "check" accent), the elements of which in turn are $4 \times 4$ Nambu-Gor'kov matrices in combined particle-hole (denoted by a "hat" accent) and spin space. The structure of the propagators and self energies in Keldysh-space and particle-hole space is as follows,

$$
\begin{aligned}
& \check{g}=\left(\begin{array}{cc}
\hat{g}^{R} & \hat{g}^{K} \\
0 & \hat{g}^{A}
\end{array}\right), \quad \hat{g}^{R, A}=\left(\begin{array}{cc}
g^{R, A} & f^{R, A} \\
\tilde{f}^{R, A} & \tilde{g}^{R, A}
\end{array}\right), \quad \hat{g}^{K}=\left(\begin{array}{cc}
g^{K} & f^{K} \\
-\tilde{f}^{K} & -\tilde{g}^{K}
\end{array}\right), \\
& \check{\Sigma}=\left(\begin{array}{cc}
\hat{\Sigma}^{R} & \hat{\Sigma}^{K} \\
0 & \hat{\Sigma}^{A}
\end{array}\right), \hat{\Sigma}^{R, A}=\left(\begin{array}{cc}
\Sigma^{R, A} & \Delta^{R, A} \\
\tilde{\Delta}^{R, A} & \tilde{\Sigma}^{R, A}
\end{array}\right), \hat{\Sigma}^{K}=\left(\begin{array}{cc}
\Sigma^{K} & \Delta^{K} \\
-\tilde{\Delta}^{K} & -\tilde{\Sigma}^{K}
\end{array}\right) .
\end{aligned}
$$

The elements of the $2 \times 2 \mathrm{Nambu}$-Gor'kov matrices are $2 \times 2$ matrices in spin space, e.g. $g^{R}=g_{\alpha \beta}^{R}$ with $\alpha, \beta=\{\uparrow, \downarrow\}$, and similarly for others. In 
writing Eqs. (1) and (2) we used general symmetries, which are accounted for by the "tilde" operation,

$$
\tilde{X}\left(\mathbf{p}_{F}, \mathbf{R}, E, t\right)=X\left(-\mathbf{p}_{F}, \mathbf{R},-E, t\right)^{*} .
$$

The quasiclassical Green's functions satisfy the Eilenberger-Larkin-Ovchinnikov transport equation and normalization condition

$$
\left[E \check{\tau}_{3}-\check{\Sigma}, \check{g}\right]_{\otimes}+i \mathbf{v}_{F} \cdot \nabla \check{g}=0, \quad \check{g} \otimes \check{g}=-\pi^{2} \check{1} .
$$

The noncommutative product $\otimes$ combines matrix multiplication with a convolution over the internal variables, and $\check{\tau}_{3}=\hat{\tau}_{3} \check{1}$ is a Pauli matrix in particlehole space.

The functional dependence of the quasiclassical propagator on the self energies is given in the form of self-consistency conditions. E.g for a weakcoupling, $s$-wave order parameter the condition reads

$$
\hat{\Delta}(\mathbf{R}, t)=\lambda \int_{-E_{c}}^{E_{c}} \frac{d E}{4 \pi i}\left\langle\hat{f}^{K}\left(\mathbf{p}_{F}, \mathbf{R}, E, t\right)\right\rangle_{\mathbf{p}_{F}},
$$

where $\lambda$ is the strength of the pairing interaction, and \langle\rangle$_{\mathbf{p}_{F}}$ denotes averaging over the Fermi surface. The cut-off energy $E_{c}$ is to be eliminated in favor of the transition temperature in the usual manner.

When the quasiclassical Green's function has been determined, physical quantities of interest can be calculated. E.g. the equilibrium local density of states at position $\mathbf{R}$ for quasiparticles with momentum $\mathbf{p}_{F}$ and energy $E$ (measured from the Fermi level) reads

$$
N\left(\mathbf{p}_{F}, \mathbf{R}, E\right)=N_{F} \frac{1}{-4 \pi i} \operatorname{Tr}\left[\hat{\tau}_{3} \hat{g}^{R}\left(\mathbf{p}_{F}, \mathbf{R}, E\right)-\hat{\tau}_{3} \hat{g}^{A}\left(\mathbf{p}_{F}, \mathbf{R}, E\right)\right] .
$$

where $N_{F}$ is the density of states on the Fermi surface.

For heterostructures, the above equations must still be supplemented with boundary conditions at the interfaces. As these conditions are non-trivial in quasiclassical theory, we present these conditions in the following chapter in detail.

\subsection{Boundary Conditions}

In order to formulate the boundary conditions at an interface between two materials, we define first an auxiliary propagator on each side of the interface [4]. Then we relate this auxiliary propagator to the full propagator via a transfer matrix. This approach is completely equivalent to a full scattering matrix approach as we will show at the end of this paragraph. However, the repeated Andreev scattering processes across the interface complicate the full scattering matrix approach, and we found the current method much easier to implement and numerically more stable $[5,6]$. 
We use for the auxiliary propagator the notation $g_{o}^{\alpha, 0}$ and $g_{i}^{\alpha, 0}$, where the upper index $\alpha=\{l, r\}$ determines the side of the interface (left or right). The lower index denotes the direction of the Fermi velocity. Incoming momenta (index $i$ ) are those with a Fermi velocity pointing towards the interface, and outgoing momenta (index $o$ ) are those with a Fermi velocity pointing away from the interface. We formulate here the boundary conditions for clean surfaces, which conserve the parallel component of the Fermi momentum, $\mathbf{p}_{\|}$. The auxiliary propagators as function of $\mathbf{p}_{\|}$, energy $E$, and time $t$ are solutions of the quasiclassical transport equation with the exact self energies, together with the normalization condition, however subject to the auxiliary boundary conditions,

$$
\check{g}_{o}^{\alpha, 0}\left(\mathbf{p}_{\|}, E, t\right)=\hat{S}_{o i}^{\alpha}\left(\mathbf{p}_{\|}\right) \check{g}_{i}^{\alpha, 0}\left(\mathbf{p}_{\|}, E, t\right) \hat{S}_{i o}^{\alpha}\left(\mathbf{p}_{\|}\right),
$$

where the following symmetries hold,

$$
\hat{S}_{i o}^{\alpha}\left(\mathbf{p}_{\|}\right)=\hat{S}_{o i}^{\alpha}\left(\mathbf{p}_{\|}\right)^{\dagger}=\hat{S}_{o i}^{\alpha}\left(\mathbf{p}_{\|}\right)^{-1} .
$$

These boundary conditions are formally equivalent to boundary conditions for an impenetrable interface, however with a surface scattering matrix $\hat{S}_{o i}^{\alpha}$ determined from the reflection amplitudes of the full scattering matrix as explained below. Once these auxiliary propagators are obtained, the full propagators can be obtained directly, without further solving the transport equation, in the following way. We define hopping amplitudes $\hat{\tau}_{i o}^{l r}, \hat{\tau}_{i o}^{r l}, \hat{\tau}_{o i}^{l r}$ and $\hat{\tau}_{o i}^{r l}$, for the four channels (left incoming to right outgoing, right incoming to left outgoing, left outgoing to right incoming, and right outgoing to left incoming) which conserve the momentum component parallel to the interface, $\mathbf{p}_{\|}$. With the help of these amplitudes we solve for the transfer matrices, $\check{t}_{i}^{\alpha}$, for incoming trajectories from the following equations,

$$
\begin{aligned}
\check{t}_{i}^{\alpha}\left(\mathbf{p}_{\|}, E, t\right) & =\hat{\tau}_{i o}^{\alpha \beta}\left(\mathbf{p}_{\|}\right) \check{g}_{o}^{\beta, 0}\left(\mathbf{p}_{\|}, E, t\right) \hat{\tau}_{o i}^{\beta \alpha}\left(\mathbf{p}_{\|}\right) \\
& \otimes\left(\check{1}+\check{g}_{i}^{\alpha, 0}\left(\mathbf{p}_{\|}, E, t\right) \otimes \check{t}_{i}^{\alpha}\left(\mathbf{p}_{\|}, E, t\right)\right),
\end{aligned}
$$

where $(\alpha \beta)=\{(l, r),(r, l)\}$. The corresponding transfer matrices for outgoing trajectories are related to the ones for incoming trajectories through the relations

$$
\check{t}_{o}^{\alpha}\left(\mathbf{p}_{\|}, E, t\right)=\hat{S}_{o i}^{\alpha}\left(\mathbf{p}_{\|}\right) \check{t}_{i}^{\alpha}\left(\mathbf{p}_{\|}, E, t\right) \hat{S}_{i o}^{\alpha}\left(\mathbf{p}_{\|}\right) .
$$

Particle conservation requires certain symmetries between the hopping elements, which are,

$$
\hat{\tau}_{o i}^{\beta \alpha}\left(\mathbf{p}_{\|}\right)=\hat{\tau}_{i o}^{\alpha \beta}\left(\mathbf{p}_{\|}\right)^{\dagger}=\hat{S}_{o i}^{\beta}\left(\mathbf{p}_{\|}\right) \hat{\tau}_{i o}^{\beta \alpha}\left(\mathbf{p}_{\|}\right) \hat{S}_{o i}^{\alpha}\left(\mathbf{p}_{\|}\right) .
$$

Consequently, only one amplitude, e.g. $\hat{\tau}_{i o}^{l r}$, contains free material parameters, the other three amplitudes depend on it. The hopping amplitudes are defined via the transmission amplitudes of the full scattering matrix as is shown 
below. Formally, they describe the modifications of the decoupled problem due to virtual hopping processes to the opposite side.

The full propagators, fulfilling the desired boundary conditions at the interface, can now be easily calculated. For incoming trajectories they are obtained from

$$
\begin{aligned}
& \check{g}_{i}^{\alpha}\left(\mathbf{p}_{\|}, E, t\right)=\check{g}_{i}^{\alpha, 0}\left(\mathbf{p}_{\|}, E, t\right) \\
+ & \left(\check{g}_{i}^{\alpha, 0}\left(\mathbf{p}_{\|}, E, t\right)+i \pi \tilde{1}\right) \otimes \check{t}_{i}^{\alpha}\left(\mathbf{p}_{\|}, E, t\right) \otimes\left(\check{g}_{i}^{\alpha, 0}\left(\mathbf{p}_{\|}, E, t\right)-i \pi \check{1}\right),
\end{aligned}
$$

and for outgoing trajectories from

$$
\begin{aligned}
& \check{g}_{o}^{\alpha}\left(\mathbf{p}_{\|}, E, t\right)=\check{g}_{o}^{\alpha, 0}\left(\mathbf{p}_{\|}, E, t\right) \\
+ & \left(\check{g}_{o}^{\alpha, 0}\left(\mathbf{p}_{\|}, E, t\right)-i \pi \check{1}\right) \otimes \check{t}_{o}^{\alpha}\left(\mathbf{p}_{\|}, E, t\right) \otimes\left(\check{g}_{o}^{\alpha, 0}\left(\mathbf{p}_{\|}, E, t\right)+i \pi \check{1}\right) .
\end{aligned}
$$

In this formulation, the boundary problem effectively reduces to calculating the auxiliary Green's functions for perfectly reflecting interfaces. Numerically this is an extremely simple task, e.g., employing the procedure of Riccati parameterization as explained below. Afterwards the boundary Green's functions for the partially transmitting interface can be obtained directly from Eqs. (12) and (13), since solving for the necessary transfer matrices (9) only involves a matrix inversion.

In the transfer-matrix description, the phenomenological parameters containing the microscopic information of the interface are the two surface scattering matrices $S_{o i}^{l, r}$ and the hopping amplitude $\tau_{i o}^{l r}$. All three quantities are $2 \times 2$ matrices in spin space. As $S_{i o}^{\alpha}$ is unitary, it depends apart from the direction of the quantization axis on two parameters, a scalar scattering phase and a spin mixing angle (or, equivalently, a spin rotation angle). Similarly, as $\tau_{i o}^{l r}$ is hermitian, it also depends on two parameters, one describing spin conserving transmission and the other spin flip transmission. All remaining quantities defined above are related to these material parameters by symmetries.

The particle-hole structures of the surface scattering matrix and the hopping amplitude are given by,

$$
\hat{S}_{o i}^{\alpha}\left(\mathbf{p}_{\|}\right)=\left(\begin{array}{cc}
S_{o i}^{\alpha}\left(\mathbf{p}_{\|}\right) & 0 \\
0 & \tilde{S}_{o i}^{\alpha}\left(\mathbf{p}_{\|}\right)
\end{array}\right), \quad \hat{\tau}_{i o}^{\alpha, \beta}\left(\mathbf{p}_{\|}\right)=\left(\begin{array}{cc}
\tau_{i o}^{\alpha \beta}\left(\mathbf{p}_{\|}\right) & 0 \\
0 & \tilde{\tau}_{i o}^{\alpha \beta}\left(\mathbf{p}_{\|}\right)
\end{array}\right),
$$

with the hole components,

$$
\begin{aligned}
\tilde{S}_{o i}^{\alpha}\left(\mathbf{p}_{\|}\right) & =S_{i o}^{\alpha}\left(-\mathbf{p}_{\|}\right)^{*}=S_{o i}^{\alpha}\left(-\mathbf{p}_{\|}\right)^{t r} \\
\tilde{\tau}_{i o}^{\alpha \beta}\left(\mathbf{p}_{\|}\right) & =\tau_{o i}^{\alpha \beta}\left(-\mathbf{p}_{\|}\right)^{*}=S_{o i}^{\alpha}\left(-\mathbf{p}_{\|}\right)^{*} \tau_{i o}^{\alpha \beta}\left(-\mathbf{p}_{\|}\right)^{*} S_{o i}^{\beta}\left(-\mathbf{p}_{\|}\right)^{*} .
\end{aligned}
$$

Finally, we relate the parameters $S_{o i}^{\alpha}$ and $\tau_{i o}^{\alpha \beta}$ to the full normal state scattering matrix $\hat{\mathbf{S}}$,

$$
\hat{\mathbf{S}}=\left(\begin{array}{cc}
\hat{\mathbf{S}}_{l l} & \hat{\mathbf{S}}_{l r} \\
\hat{\mathbf{S}}_{r l} & -\hat{\mathbf{S}}_{r r}
\end{array}\right)
$$


The scattering matrix is diagonal in particle-hole space, with diagonal components

$$
\hat{\mathbf{S}}_{\alpha \alpha}=\left(1+\pi^{2} \hat{\tau}_{o i}^{\alpha \beta} \hat{\tau}_{i o}^{\beta \alpha}\right)^{-1}\left(1-\pi^{2} \hat{\tau}_{o i}^{\alpha \beta} \hat{\tau}_{i o}^{\beta \alpha}\right) \hat{S}_{o i}^{\alpha},
$$

and off-diagonal components

$$
\hat{\mathbf{S}}_{\alpha \beta}=\left(1+\pi^{2} \hat{\tau}_{o i}^{\alpha \beta} \hat{\tau}_{i o}^{\beta \alpha}\right)^{-1} 2 \pi \hat{\tau}_{o i}^{\alpha \beta} .
$$

These identities serve as a precise definition of the auxilary parameters of the theory, $S_{o i}^{l}\left(\mathbf{p}_{\|}\right), S_{o i}^{r}\left(\mathbf{p}_{\|}\right)$, and $\tau_{i o}^{l r}\left(\mathbf{p}_{\|}\right)$in terms of the physical parameters of the full scattering matrix.

\subsection{Riccati parameterization}

The method of the Riccati parameterization [7-9] of the quasiclassical Green's functions has proved powerful during recent years. It accounts automatically for the normalization condition. A corresponding parameterization for distribution functions was applied to the Keldysh part of the Green's functions. The combined equations in their general form are [9]

$$
\begin{aligned}
& \hat{g}^{K}=-2 i \pi \hat{N}^{R} \otimes\left(\begin{array}{cc}
\left(x-\gamma^{R} \otimes \tilde{x} \otimes \tilde{\gamma}^{A}\right) & -\left(\gamma^{R} \otimes \tilde{x}-x \otimes \gamma^{A}\right) \\
-\left(\tilde{\gamma}^{R} \otimes x-\tilde{x} \otimes \tilde{\gamma}^{A}\right) & \left(\tilde{x}-\tilde{\gamma}^{R} \otimes x \otimes \gamma^{A}\right)
\end{array}\right) \otimes \hat{N}^{A}, \\
& \hat{g}^{R, A}= \pm i \pi \hat{N}^{R, A} \otimes\left(\begin{array}{cc}
\left(1+\gamma^{R, A} \otimes \tilde{\gamma}^{R, A}\right) & 2 \gamma^{R, A} \\
-2 \tilde{\gamma}^{R, A} & -\left(1+\tilde{\gamma}^{R, A} \otimes \gamma^{R, A}\right)
\end{array}\right)
\end{aligned}
$$

with

$$
\hat{N}^{R, A}=\left(\begin{array}{cc}
\left(1-\gamma^{R, A} \otimes \tilde{\gamma}^{R, A}\right)^{-1} & 0 \\
0 & \left(1-\tilde{\gamma}^{R, A} \otimes \gamma^{R, A}\right)^{-1}
\end{array}\right) .
$$

Thus, the problem is reduced to the solution for two $2 \times 2$ matrices in spin space, $\gamma^{R}$ and $x$. Using fundamental symmetries (particle-hole, retardedadvanced) like Eq. (3) and

$$
\gamma^{A}\left(\mathbf{p}_{F}, \mathbf{R}, E, t\right)=\gamma^{R}\left(-\mathbf{p}_{F}, \mathbf{R},-E, t\right)^{t r},
$$

where $t r$ denotes the spin-matrix transpose operation, the full retarded, advanced and Keldysh Green's functions are obtained from $\gamma^{R}$ and $x$. The transport equations for the functions $\gamma^{R}\left(\mathbf{p}_{F}, \mathbf{R}, E, t\right)$ and $x\left(\mathbf{p}_{F}, \mathbf{R}, E, t\right)$ are

$$
2 E \gamma^{R}+i \mathbf{v}_{F} \nabla \gamma^{R}=\gamma^{R} \otimes \tilde{\Delta}^{R} \otimes \gamma^{R}+\Sigma^{R} \otimes \gamma^{R}-\gamma^{R} \otimes \tilde{\Sigma}^{R}-\Delta^{R},
$$

and

$$
\begin{aligned}
i \partial_{t} x+i \mathbf{v}_{F} \nabla x & +\left(-\gamma^{R} \otimes \tilde{\Delta}^{R}-\Sigma^{R}\right) \otimes x+x \otimes\left(-\Delta^{A} \otimes \tilde{\gamma}^{A}+\Sigma^{A}\right) \\
& =-\gamma^{R} \otimes \tilde{\Sigma}^{K} \otimes \tilde{\gamma}^{A}+\Delta^{K} \otimes \tilde{\gamma}^{A}+\gamma^{R} \otimes \tilde{\Delta}^{K}-\Sigma^{K}
\end{aligned}
$$




\section{Results: Ballistic Case}

As an example we consider here the Andreev quasiparticle spectrum in a half-metallic ferromagnet between two singlet superconductors. This corresponds to the device shown on the left side in Fig. 1. In this case, on the half-metallic side only quasiparticles with one spin direction with respect to the quantization axis are itinerant. The stable equilibrium configuration of such a system is a $\pi$-junction, in which the phase of the singlet order parameter on both sides of the interface differs by $\pi$ [5]. Characteristic of such a heterostructure is the presence of triplet correlations near the interface. Shown in Fig. 2 are the singlet order parameter and all three triplet compo-

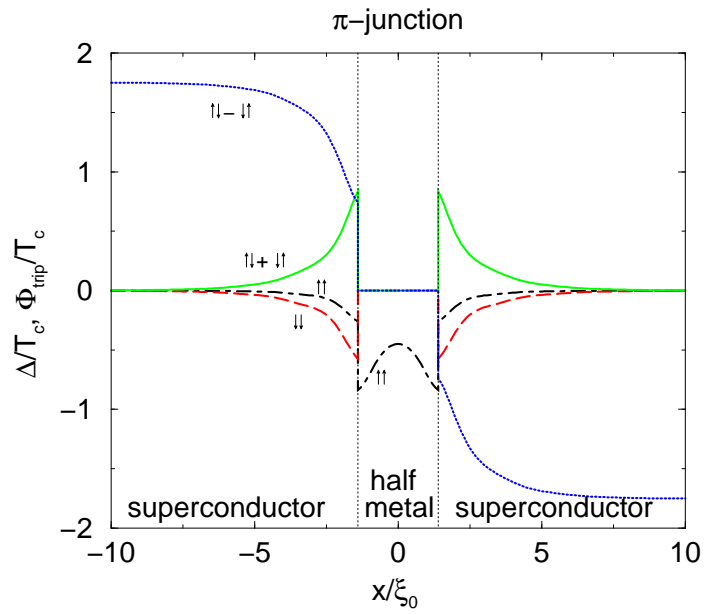

Fig. 2. Self consistent order parameter and triplet correlations in an $\mathrm{S} / \mathrm{HM} / \mathrm{S} \pi$ junction. The calculations are for temperature $T=0.05 T_{c}$, and for $\tau_{\downarrow, \uparrow} / \tau_{\uparrow, \uparrow}=0.7$

nents which are induced near the interface by the spin rotation effect [10]. The spin rotation effect is taken into account via a surface scattering ma$\operatorname{trix} \hat{S}_{o i}=\exp \left(i \theta \sigma_{z} / 2\right) \hat{1}$ at the superconducting side of the interface, where $\theta$ defines a spin-rotation angle and $\sigma_{z}$ denotes the Pauli spin matrix $[10,11]$. Generally, the value of $\theta$ depends on the angle of impact, $\psi$ [10] and can approach values of the order of $\pi$ for strong band splitting [12]. For definiteness, we present results for $\theta=0.75 \pi \cos \psi$. On the half-metallic side the scattering matrix has no spin structure. Further, we use the hopping amplitude $\tau_{i o}=\left(1+S_{i o}\right) \tau_{0} \cos \psi$, where $\tau_{0}=\left(\tau_{\uparrow, \uparrow}, \tau_{\downarrow, \uparrow}\right)^{\mathrm{T}}$ is determined by the two spin scattering channels from the superconductor to the half-metallic spinup band. This reflects a spin rotation during transmission which is half of the spin rotation during reflection. The $\cos \psi$ factor accounts for the reduced transmission at large impact angles. We present results for $\tau_{\downarrow, \uparrow} / \tau_{\uparrow, \uparrow}=0.7$ and $0.1,2 \pi \tau \uparrow, \uparrow=1.0$. The length of the half metallic region is $3 \xi_{0}$ with the coherence length $\xi_{0}=v_{f} / 2 \pi T_{c}$. We assume cylindrical Fermi surfaces. 
We have studied the behavior of such a heterostructure as a Josephson device. If a phase difference $\phi$ in the order parameter is present between the left and right end of the heterostructure, the quasiparticle spectrum in the half metal is modified. In Figs. 3 and 4 we show results of our calculations for two sets of parameters.

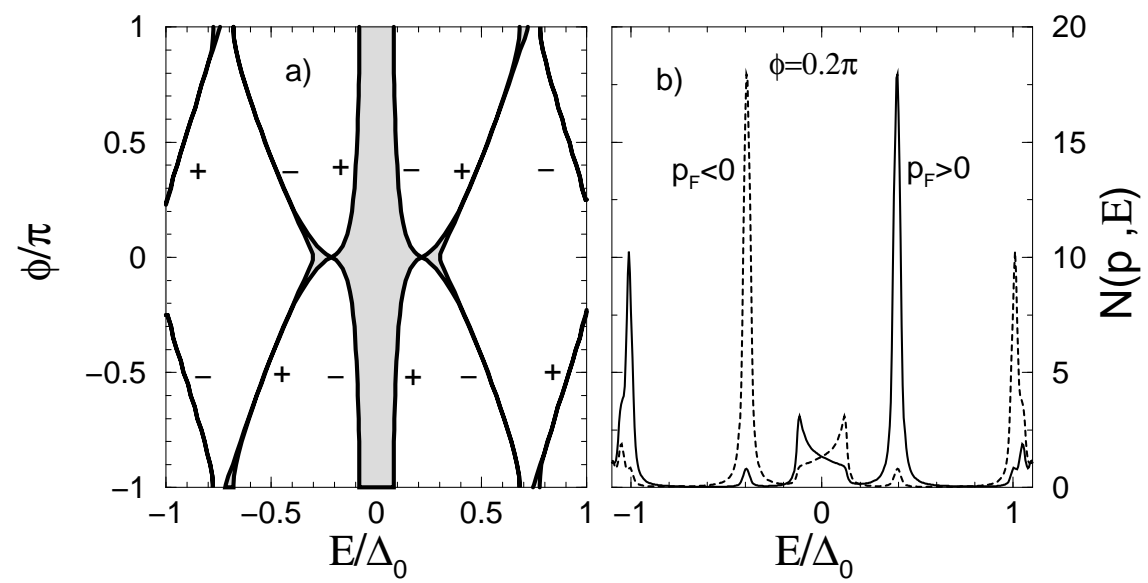

Fig. 3. Density of states at $T=0.05 T_{c}$ for quasiparticles with normal impact at the half-metallic side of the left interface, for $\tau_{\downarrow, \uparrow} / \tau_{\uparrow, \uparrow}=0.7$. a) Dispersion of the maxima of the density of states as function of phase difference. Gapped regions are white, ungapped regions gray. The signs indicate the direction of the current carried by the Andreev states. b) As an example we show spectra for a selected phase difference

The spectra consist out of Andreev quasiparticle bands, separated by gaps. In Figs. 3 a) and 4 a) we show these gapped-regions (white) and the nongapped regions (grey) of the density of states at the half-metallic side of the left interface as a function of the phase difference. One characteristic spectrum for fixed phase difference for both positive and negative momentum direction is shown in Figs. $3 \mathrm{~b}$ ) and $4 \mathrm{~b}$ ). The dispersion of the maxima in the density of states with phase difference determines the sign of the Josephson current trough the device. We indicate in Figs. 3a) and 4a) by + and - Andreev states, which carry current in positive and negative direction respectively. It can be seen that the sign of the Josephson current corresponds to the sign of $d E(\phi) / d \phi$, where $E(\phi)$ denotes the dispersion of the maxima in the density of states. This is analogous to the current carried by a bound state $E_{b}$ dispersing with phase $\phi$, which is given by $J_{b} \sim\left(d E_{b} / d \phi\right) n\left(E_{b}\right)$, where $n\left(E_{b}\right)$ is the equilibrium fermion distribution function. 


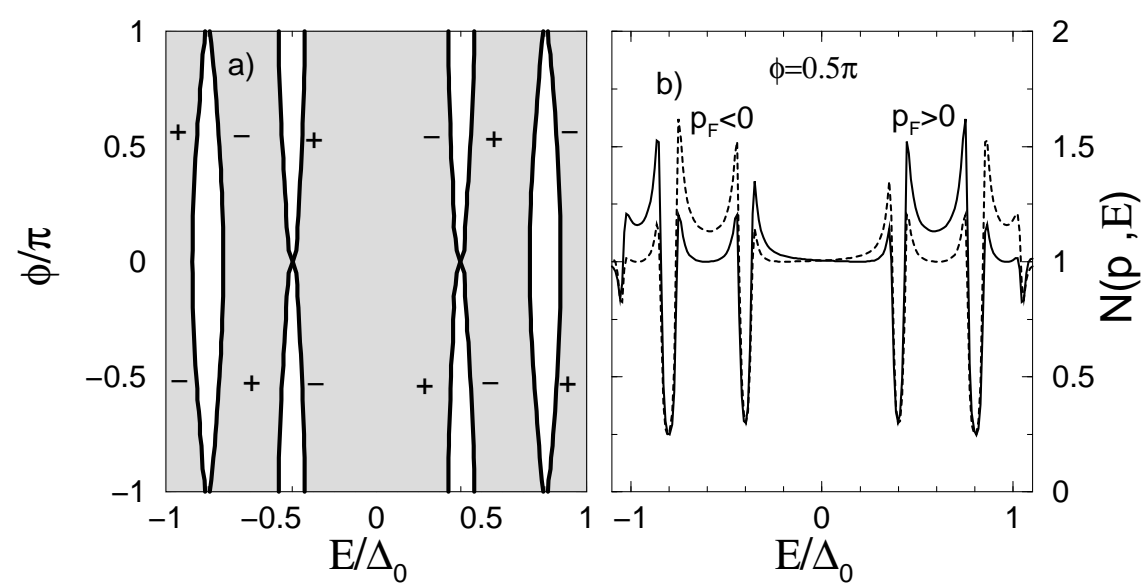

Fig. 4. The same as Fig. 3 for $\tau_{\downarrow, \uparrow} / \tau_{\uparrow, \uparrow}=0.1$

\section{Basic Equations: Diffusive Case}

\subsection{Transport Equations}

The fundamental quantity for diffusive transport is the Usadel Green's function [13], which is the momentum average of the quasiclassical Green's function $\check{g}(\mathbf{R}, E, t)=\left\langle\check{g}\left(\mathbf{p}_{F}, \mathbf{R}, E, t\right)\right\rangle_{\mathbf{p}_{F}}$. It is a functional of momentum averaged self energies $\check{\Sigma}(\mathbf{R}, E, t)=\left\langle\check{\Sigma}\left(\mathbf{p}_{F}, \mathbf{R}, E, t\right)\right\rangle_{\mathbf{p}_{F}}$. The structures of $\check{g}$ and $\check{\Sigma}$ are the same as in Eqs. (1) and (2). Eq. (3) is replaced by

$$
\tilde{X}(\mathbf{R}, E, t)=X(\mathbf{R},-E, t)^{*} .
$$

The Usadel Green's function obeys the following transport equation and normalization condition [13],

$$
\left[E \hat{\tau}_{3} \check{1}-\check{\Sigma}, \check{g}\right]_{\otimes}+\frac{D}{\pi} \nabla_{j}\left(\check{g} \otimes \nabla_{j} \check{g}\right)=0, \quad \check{g} \otimes \check{g}=-\pi^{2} \check{1} .
$$

Here, summation over the repeated index $j$ is implied. $\check{\Sigma}$ does not contain the non-magnetic impurity scattering self energy anymore. We will solve these equations by using the Riccati parameterization technique and present below the resulting equations for the diffusive case. The corresponding equations in a finite vector potential are obtained by applying a gauge transformation to Eqs. (26) (and to Eqs. (28), (29) below).

\subsection{Boundary Conditions}

In the diffusive limit the boundary conditions are formulated in terms of the momentum averaged transfer matrices and Green's functions. The resulting 
boundary conditions for spin-conserving interfaces were found by Nazarov [14] and follow from the transfer-matrix approach outlined above [6]. They are

$$
\begin{aligned}
& \sigma_{\beta} \check{g}^{\beta} \frac{d}{d z} \check{g}^{\beta}=\frac{1}{S R_{b}} \frac{2 \pi^{2} T\left[\check{g}^{\beta}, \check{g}^{\alpha}\right]}{4 \pi^{2}-T\left(\left\{\check{g}^{\beta}, \check{g}^{\alpha}\right\}+2 \pi^{2}\right)} \\
& \sigma_{\beta} \check{g}^{\beta} \frac{d}{d z} \check{g}^{\beta}=\sigma_{\alpha} \check{g}^{\alpha} \frac{d}{d z} \check{g}^{\alpha},
\end{aligned}
$$

where $z$ is the coordinate along the interface normal, $\sigma_{\alpha(\beta)}$ and $\check{g}^{\alpha(\beta)}$ refers to the conductivity and the Keldysh Green's function on side $\alpha(\beta)$ of the interface, $S$ is the surface area of the contact, $R_{b}$ the boundary resistance and $T$ the transmission probability, $T=\frac{4 \pi^{2}|\tau|^{2}}{\left(1+\pi^{2}|\tau|^{2}\right)^{2}}$.

\subsection{Riccati parameterization}

We use the same parameterization, Eqs. (20)-(21), for the momentum averaged Green's functions. Note that the such defined diffusive quantities $\gamma^{R}(\mathbf{R}, E, t)$ and $x(\mathbf{R}, E, t)$ are by no means in a simple way related to the ballistic quantities $\gamma^{R}\left(\mathbf{p}_{F}, \mathbf{R}, E, t\right)$ and $x\left(\mathbf{p}_{F}, \mathbf{R}, E, t\right)$. Consequently, also the structure of the transport equations is very different. In particular the gradient terms in the transport equations for these quantities are modified with respect to the gradient terms in the transport equations for the ballistic case. Instead of Eqs. (23) and (24) we obtain for the diffusive case

$$
\begin{aligned}
2 E \gamma^{R} & -i D\left[\nabla^{2} \gamma^{R}+\left(\nabla_{j} \gamma^{R}\right) \otimes \frac{\tilde{f}^{R}}{i \pi} \otimes\left(\nabla_{j} \gamma^{R}\right)\right] \\
& =\gamma^{R} \otimes \tilde{\Delta}^{R} \otimes \gamma^{R}+\Sigma^{R} \otimes \gamma^{R}-\gamma^{R} \otimes \tilde{\Sigma}^{R}-\Delta^{R},
\end{aligned}
$$

and

$$
\begin{aligned}
i \partial_{t} x & -i D\left[\nabla^{2} x-\left(\nabla_{j} \gamma^{R}\right) \otimes \frac{\tilde{g}^{K}}{i \pi} \otimes\left(\nabla_{j} \tilde{\gamma}^{A}\right)\right. \\
& \left.+\left(\nabla_{j} \gamma^{R}\right) \otimes \frac{\tilde{f}^{R}}{i \pi} \otimes\left(\nabla_{j} x\right)+\left(\nabla_{j} x\right) \otimes \frac{f^{A}}{i \pi} \otimes\left(\nabla_{j} \tilde{\gamma}^{A}\right)\right] \\
& +\left(-\gamma^{R} \otimes \tilde{\Delta}^{R}-\Sigma^{R}\right) \otimes x+x \otimes\left(-\Delta^{A} \otimes \tilde{\gamma}^{A}+\Sigma^{A}\right) \\
& =-\gamma^{R} \otimes \tilde{\Sigma}^{K} \otimes \tilde{\gamma}^{A}+\Delta^{K} \otimes \tilde{\gamma}^{A}+\gamma^{R} \otimes \tilde{\Delta}^{K}-\Sigma^{K} .
\end{aligned}
$$

Summation over the repeated index $j$ is implied. The expressions for $\tilde{f}^{R}, f^{A}$, and $\tilde{g}^{K}$ are obtained by comparing Eq. (1) with Eqs. (20)-(21).

\section{Results: Diffusive Case}

As an example we study for this case the heterostructure shown on the right side of Fig. 1, which contains a weak diffusive ferromagnet between two superconductors. A domain wall of Bloch type is centered in the ferromagnetic 
part. Problems involving domain walls have previously been treated only with techniques that do not allow access to information on spatial variations, [15] or elaborate methods involving rotating coordinate systems.[16] However, since the Riccati description contains the full $2 \times 2$ spin structure, it extends easily to account for any such situation.

We adopt for the description of a weak ferromagnetic materials ( $J$ much smaller than the Fermi energy) a quasiparticle dispersion given by a spindependent energy shift $E \hat{\tau}_{3} \rightarrow E \hat{\tau}_{3}-\boldsymbol{J}(\mathbf{R}) \cdot \hat{\boldsymbol{\sigma}}$, in Eq. (26). Here $\boldsymbol{J}(\mathbf{R})$ denotes the effective exchange field of the ferromagnet, and $\boldsymbol{\sigma}$ denotes the vector of Pauli spin matrices. We solve the Usadel equations using the Riccati parameterization for a ferromagnetic material, which contains a domain wall of the Bloch type. We model the domain wall by a $\boldsymbol{J}$-vector rotating across the domain wall with the polar angle $\theta_{J}=\operatorname{atan}\left(z / d_{W}\right)$, where $d_{W}$ parameterizes the width of the domain wall.

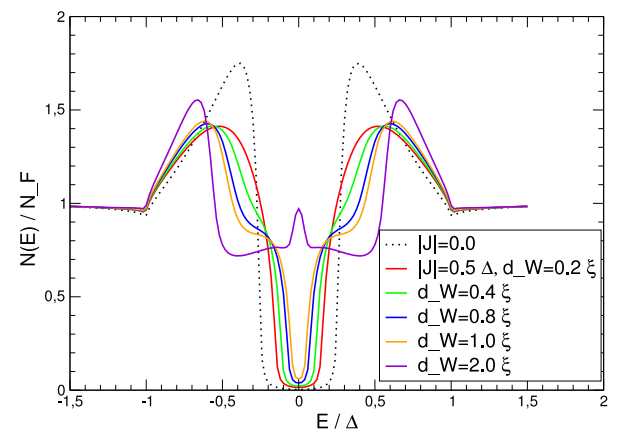

Fig. 5. Local density of states as a function of energy in the center of a heterostructure containing a weak ferromagnet $(J=0.5 \Delta)$ of length $d_{F}=2 \xi_{0}$ between two superconductors. A Bloch domain wall of length $d_{W}$ is centered in the ferromagnet (see Fig. 1). Results for various $d_{W}$ are shown

In Fig. 5 we present results for the local density of states at the center position of the heterostructure shown in Fig 1 . The length of the ferromagnet is $d_{F}=2 \xi_{0}$, where $\xi_{0}=\sqrt{D / \Delta}$ is the coherence length in the ferromagnet, and $\Delta$ is the gap in the superconductors. The dotted curve shows the behavior for a normal metal between two superconductors, showing the well known minigap. The effect of a domain wall in the center of the ferromagnet is shown for several wall thicknesses $d_{W}$ in Fig. 5. The new structures in the presence of the domain wall in Fig. 5 are due to spin-triplet correlations which mix between all three spin-triplet channels due to the continuous variation of the spin quantization axis across the domain wall [16]. This leads to the appearence of additional Andreev bound states inside the minigap, modifying the total density of states. In general, the presence of the domain wall reduces the minigap, and for thick enough domain walls the minigap closes completely. This suggests that the width of the domain walls can influence the transport properties of such devices considerably. 


\section{Conclusions}

We have developed a framework for studying heterostructures with superconducting and ferromagnetic parts, and have applied it to two cases: a ferromagnet in the ballistic regime with strong spin polarization $\left(J \sim E_{F}\right)$; and a weak ferromagnet $\left(J \ll E_{F}\right)$ in the diffusive regime, having an inhomogeneous, non-collinear spin magnetization (Bloch domain wall). In both cases we have studied the superconducting proximity effect with the ferromagnet sandwiched between two superconductors. We have shown, that singlet-triplet mixing occurs near interfaces between superconductors and strong ferromagnets, or near domain-wall structures in the case of weak ferromagnets in proximity to a superconductor. The origin of equal-spin triplet correlations in the two studied cases is very different. In a strong ferromagnet between two superconductors, the singlet triplet mixing takes place within the superconductor, in a layer of the order of the coherence length near the interface, enabling triplet correlations to penetrate into the ferromagnet. In contrast, in weak ferromagnets with domain walls of Bloch type, equal-spin triplet correlations are produced within the ferromagnet, near the domain walls.

\section{Acknowledgements}

This work was supported by Deutsche Forschungsgemeinschaft within the Center for Functional Nanostructures.

\section{References}

1. G. Eilenberger, Z. Phys. 214, 195 (1968).

2. A. I. Larkin and Y. N. Ovchinnikov, Sov. Phys. JETP 28, 1200 (1969).

3. J. W. Serene and D. Rainer, Phys. Rep. 101, 221 (1983).

4. J. C. Cuevas, A. Martín-Rodero, and A. Levy Yeyati, Phys. Rev. B 54, 7366 (1996); J. C. Cuevas and M. Fogelström, Phys. Rev. B 64, 104502 (2001).

5. M. Eschrig, J. Kopu, J. C. Cuevas, and Gerd Schön, Phys. Rev. Lett. 90, 137003 (2003).

6. J. Kopu, M. Eschrig, J. C. Cuevas, and M. Fogelström, Phys. Rev. B 69, 094501 (2004).

7. Y. Nagato, K. Nagai and J. Hara, J. Low Temp. Phys. 93, 33 (1993); S. Higashitani and K. Nagai, J. Phys. Soc. Jpn. 64, 549 (1995).

8. N. Schopohl and K. Maki, Phys. Rev. B 52, 490 (1995).

9. M. Eschrig, Phys. Rev. B, 61, 9061 (2000).

10. T. Tokuyasu, J.A. Sauls, and D. Rainer, Phys. Rev. B 38, 8823 (1988).

11. M. Fogelström, Phys. Rev. B 62, 11812 (2000).

12. Yu.S. Barash and I.V. Bobkova, Phys. Rev. B 65, 144502 (2002).

13. K. D. Usadel, Phys. Rev. Lett. 25, 507 (1977).

14. Yu. V. Nazarov, Superlatt. and Microstruct. 25, 121 (1999).

15. N. M. Chtchelkatchev and I. S. Burmistrov, Phys. Rev. B 68, 140501(R) (2003).

16. F. S. Bergeret, A. F. Volkov, and K. B. Efetov, Phys. Rev. Lett. B 86, 4096 (2001); R. Mélin and S. Peysson, Phys. Rev. B 68, 174515 (2003). 